\title{
Resveratrol mediates cell cycle arrest and cell death in human esophageal squamous cell carcinoma by directly targeting the EGFR signaling pathway
}

\author{
ZIXUAN JIN ${ }^{1}$, WEI FENG ${ }^{2}$, YING JI ${ }^{2}$ and LONGYU JIN ${ }^{2}$ \\ ${ }^{1}$ Department of Biochemistry, The High School Attached To Hunan Normal University, Changsha, Hunan 410006; \\ ${ }^{2}$ Department of Cardiothoracic Surgery, The Third Xiangya Hospital, Central South University, \\ Changsha, Hunan 410013, P.R. China
}

Received August 13, 2015; Accepted August 25, 2016

DOI: $10.3892 / 01.2016 .5391$

\begin{abstract}
Resveratrol is a small polyphenol that has been intensively studied in a wide spectrum of therapeutic fields. More recently, resveratrol has been demonstrated to exert its antitumor activity in numerous tumor models. The present study reported that resveratrol exhibited a marked anti-proliferative effect on human esophageal squamous cell carcinoma (ESCC) cells by inducing cell cycle $\mathrm{G}_{0} / \mathrm{G}_{1}$ phase arrest and cell death, which was associated with a decrease in the expression levels of cyclin D1 and an increase in cleaved PARP/cleaved caspase-3 expression levels. The mechanisms underlying the antitumor potency of resveratrol were principally attributed to the downregulation of epidermal growth factor receptor (EGFR) signaling. The western blotting results showed that exposure of ESCC cells to resveratrol inhibited EGF-induced EGFR activation in addition to decreasing the total protein levels of EGFR and membrane/nuclear localization. In summary, the results suggested that resveratrol, or an associated analog, may have a role in the management of human ESCC.
\end{abstract}

\section{Introduction}

Human esophageal squamous cell carcinoma (ESCC) is the 6th leading cause of cancer-associated mortality, and the 8 th most common type of cancer worldwide (1). ESCC

Correspondence to: Professor Longyu Jin, Department of Cardiothoracic Surgery, The Third Xiangya Hospital, Central South University, 138 Tongzipo Road, Yuelu, Changsha, Hunan 410013, P.R. China

E-mail: jinlongyu1123@163.com

Abbreviations: EGFR, epidermal growth factor receptor; ESCC, esophageal squamous cell carcinoma

Key words: esophageal squamous cell carcinoma, resveratrol, epidermal growth factor receptor is the most common histology of esophageal cancers, and has a high incidence in China, South Africa, France and Italy (2). Epidemiological and etiological studies have previously demonstrated that certain environmental and genetic factors have crucial roles in esophageal carcinogenesis (3-7). Although there have been advances in the fields of surgery and chemotherapy, the prognosis for ESCC is typically poor as the majority of patients diagnosed are elderly and present with advanced-stage disease, rendering them unsuitable for curative resection and maintaining the five-year survival rate for ESCC at $<15 \%$ (1). Therefore, improving the current understanding of the molecular mechanisms underlying ESCC pathogenesis and identifying effective therapeutic agents are required to facilitate the development of novel therapies for this disease.

Resveratrol is present in red grapes, wine, nuts and common garden plants, and is one of the most intensively investigated of all the phytochemicals with putative beneficial effects on human health (8). The effects of resveratrol include anti-oxidant and anti-aging properties, improvement of insulin sensitivity and reduction of cardiovascular disease risk (9-11). Previous studies have also indicated that resveratrol is able to function as a chemo preventive and chemotherapeutic agent in certain types of human carcinomas, including those of the colorectum, skin, liver, lung, pancreas, breast and prostate (8,12-14). Previous studies suggesting that a dietary pattern rich in resveratrol may reduce the risk of ESCC (15). However, the molecular mechanisms underlying resveratrol-induced antitumor effects have yet to be elucidated. A number of potential mechanisms have been suggested to be involved in resveratrol-induced cancer preventative effects, including the inhibition of tumorigenesis-associated transcription factor activation, the suppression of various protein kinases and the regulation of the expression of specific genes involved in cell cycle progression, apoptosis, angiogenesis and metastasis $(8,12,16-18)$.

The epidermal growth factor receptor (EGFR) is a receptor tyrosine kinase that mediates intracellular signaling in response to various extracellular stimuli (19). Previous studies have demonstrated that EGFR is activated and overexpressed in numerous types of human epithelial cancers, including human ESCC (19-21). The aberrant regulation of 
EGFR expression activates downstream signaling pathways, including extracellular signal-regulated kinases (ERKs) and protein kinase B (AKT), which increase tumor cell proliferation, survival and invasiveness (19). Modulation of the EGFR signaling pathway is considered to have a crucial role in cancer prevention (19). However, the role of resveratrol in the regulation of EGFR in order to mediate its carcinogenic effects has yet to be demonstrated in ESCC. In the present study, the antitumor efficacy of resveratrol in human ESCC cells and the role of EGFR in the antitumor activity of resveratrol, as well as the mechanisms underlying the inhibition of EGFR activation by resveratrol, are investigated. The results demonstrated that resveratrol exhibited a marked inhibitory effect on EGFR tyrosine kinase activity in vitro, in addition to downregulating EGFR protein levels and the expression levels of the downstream gene cyclin D1, which was also associated with resveratrol-mediated cell cycle arrest and cell death.

\section{Materials and methods}

Cell lines and culture. All cell lines were purchased from the Cell Bank of Type Culture Collection of the Chinese Academy of Sciences (Shanghai, China). KYSE150, KYSE510 and Eca109 ESCC cells were cultured in RPMI-1640 medium (Invitrogen; Thermo Fisher Scientific, Inc., Waltham, MA, USA) supplemented with $10 \%$ fetal bovine serum (Invitrogen; Thermo Fisher Scientific, Inc.), $100 \mathrm{U} / \mathrm{ml}$ penicillin and $100 \mathrm{mg} / \mathrm{ml}$ streptomycin. All cell lines were incubated at $37^{\circ} \mathrm{C}$ in a humidified atmosphere containing $5 \% \mathrm{CO}_{2}$.

Reagents and antibodies. Resveratrol was purchased from Sigma-Aldrich (Merck Millipore, Darmstadt, Germany). Sub-confluent cells were treated with the compound at various concentrations $(0,20,40$ and $80 \mu \mathrm{M})$ for $24 \mathrm{~h}$. The final concentration of dimethyl sulfoxide (DMSO) in the culture media was maintained at $<0.1 \%$, which had no significant effect on the cell growth. Anti-EGFR (\#4267; 1:2,000), anti-phosphorylated (p)-EGFR (Tyr1068; \#3777; 1:2,000), anti-p-AKT (Ser473; \#4060; 1:1,000), anti-p-ERK1/2 (Thr202/Tyr204; \#4370; 1:2,000), anti-cyclinD1 (\#2978; $1: 1,000)$, anti- $\alpha$-tubulin (\#2144; 1:5,000), cleaved PARP (\#5625; 1:1,000), cleaved caspase-3 (\#9664; 1:1,000) and anti-lamin B (\#13415; 1:2,000) antibodies were purchased from Cell Signaling Technology, Inc. (Danvers, MA, USA). Anti- $\beta$-actin (\#sc-1615; 1: 5,000), anti-rabbit immunoglobulin (Ig) G-horseradish peroxidase (HRP) (\#sc-2040; 1:10,000) and anti-mouse IgG-HRP (\#sc-2039; 1:10,000) were purchased from Santa Cruz Biotechnology (Dallas, TX, USA). Anti-N-cadherin (\#610920; 1:3,000) was purchased from BD Biosciences (San Jose, CA, USA).

Western blotting. Cells were harvested by trypsinization and centrifuged $\left(108 \times \mathrm{g}\right.$ for $5 \mathrm{~min}$ at $\left.4^{\circ} \mathrm{C}\right)$. The cell pellets were lysed in Nonidet P-40 cell lysis buffer (50 mM Tris-Cl, pH 8.0, $150 \mathrm{mM} \mathrm{NaCl}, 0.5 \%$ Nonidet P-40 and protease inhibitor mixture; Thermo Fisher Scientific, Inc.). The extracted protein concentrations were determined using a Bradford assay (Bio-Rad Laboratories, Inc., Hercules, CA, USA). Proteins were separated by sodium dodecyl sulfate polyacrylamide gel electrophoresis and electrically transferred to a polyvinylidene difluoride membrane (EMD Millipore, Billerica, MA, USA). Following blocking with 5\% nonfat dry milk in Tris-buffered saline (TBS), the membranes were hybridized to specific primary antibodies overnight at $4^{\circ} \mathrm{C}$, washed three times with TBS-Tween 20 and then incubated with the secondary antibodies conjugated with HRP for $1 \mathrm{~h}$ at room temperature. The membranes were then washed three times in TBS-Tween 20 at room temperature. Visualization of proteins was performed using the ChemiDoc XRS system with Image Lab software (Bio-Rad Laboratories, Inc.). For EGF treatment, the cells were incubated in serum-free medium overnight and then treated with resveratrol at various concentrations $(0,20$ and $40 \mu \mathrm{M})$ for $2 \mathrm{~h}$. Following stimulation with $50 \mathrm{ng} / \mathrm{ml}$ EGF (Thermo Fisher Scientific, Inc.) for $30 \mathrm{~min}$, the cells were harvested and the relative protein expression levels were determined by western blot analysis.

Subcellular proteome fractionation. The ESCC cells were treated with resveratrol $(0,20,40$ and $80 \mu \mathrm{M})$ for $48 \mathrm{~h}$, the cells were harvested by trypsinization and centrifuged (108 $\mathrm{x} \mathrm{g}$ for $5 \mathrm{~min}$ at $4^{\circ} \mathrm{C}$ ). The subcellular proteome fractions were prepared using a ProteoExtract Subcellular Proteome Extraction kit (EMD Millipore) according to the manufacturer's instructions. The subcellular proteome fractions were subjected to western blot analysis.

MTS assay. To determine the cytotoxicity of resveratrol, the cells were seeded $\left(3 \times 10^{3}\right.$ cells/well) in 96-well plates and cultured at $37^{\circ} \mathrm{C}$ in a humidified atmosphere containing $5 \%$ $\mathrm{CO}_{2}$ overnight. The cells were then cultured with fresh medium, treated with various doses of resveratrol or DMSO and cultured for various time periods $(0,24,48$ and $72 \mathrm{~h})$. The cytotoxicity of resveratrol was evaluated using an MTS assay kit (Promega Corporation, Madison, WI, USA) according to the manufacturer's instructions. The absorbance was read at $490 \mathrm{~nm}$.

Soft agar colony assay. In order to examine anchorage-independent growth, KYSE150, Eca109 and KYSE510 cells $(8,000$ cells $/ \mathrm{ml})$ were suspended in $1 \mathrm{ml} 0.3 \%$ agar with Eagle's basal medium (Thermo Fisher Scientific, Inc.) supplemented with $10 \%$ FBS, $1 \%$ antibiotics $(100 \mathrm{U} / \mathrm{ml}$ penicillin and $100 \mathrm{mg} / \mathrm{ml}$ streptomycin) and various concentrations of resveratrol $(0,20,40$ and $80 \mu \mathrm{M})$ and seeded onto 6 -well plates containing a $0.6 \%$ agar base. The cultures were maintained in a $5 \% \mathrm{CO}_{2}$ incubator at $37^{\circ} \mathrm{C}$ for $1-2$ weeks, and then the colonies were imaged under a light microscope and counted using the Image-Pro Plus software program version 6 (Media Cybernetics, Inc., Rockville, MD, USA).

Flow cytometry. Flow cytometry was used to quantify the number of cells in each phase of the cell cycle. The cells $\left(2 \times 10^{5}\right)$ were seeded into 6 -well plates and treated with various concentrations $(0,20,40,80 \mu \mathrm{M})$ of resveratrol for $24 \mathrm{~h}$. The cells were then harvested by trypsinization and centrifuged $\left(108 \times \mathrm{g}\right.$ for $5 \mathrm{~min}$ at $\left.4^{\circ} \mathrm{C}\right)$, then washed with phosphate-buffered saline (PBS) twice and fixed in $70 \%$ ethanol overnight at $4^{\circ} \mathrm{C}$. Subsequently, the cells were stained in the dark with $50 \mu \mathrm{g} / \mathrm{ml}$ propidium iodide containing $0.1 \%$ ribonuclease $\mathrm{A}$ (BD Biosciences) in $400 \mu \mathrm{l} \mathrm{PBS}$ at $25^{\circ} \mathrm{C}$ for 
A
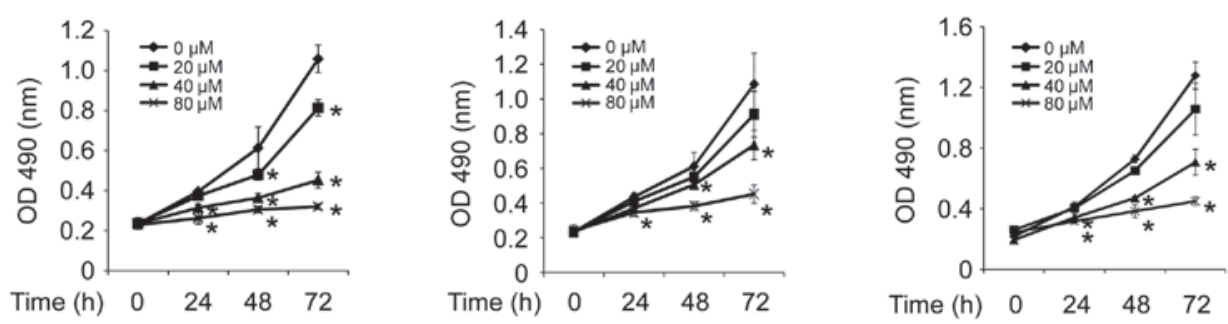

B a Resveratrol $(\mu \mathrm{M})$
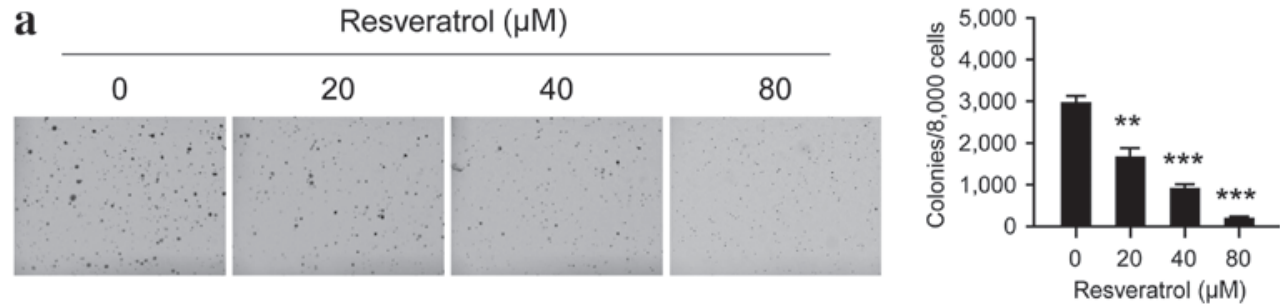

b

Resveratrol $(\mu \mathrm{M})$

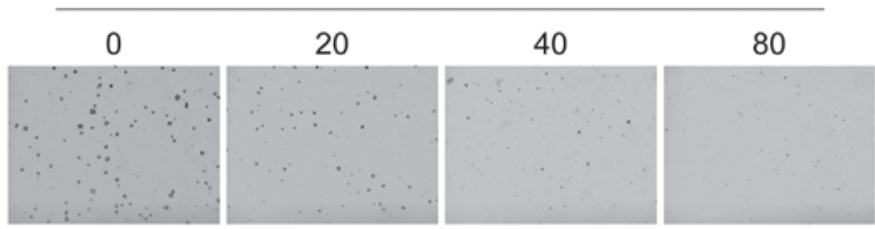

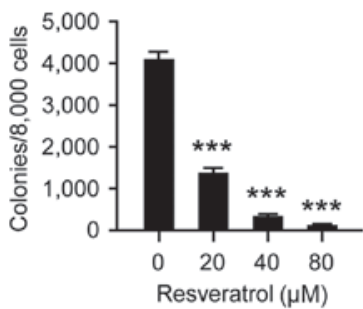

c

Resveratrol $(\mu \mathrm{M})$
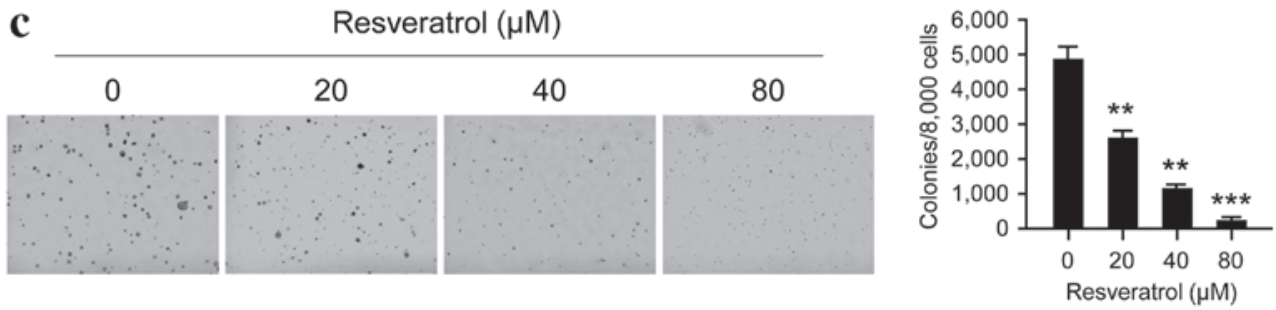

Figure 1. Inhibitory effects of resveratrol on the anchorage-dependent and -independent growth of human ESCC cells. (A) Resveratrol inhibits anchorage-dependent growth in human ESCC cells, including KYSE510 (left), Eca109 (middle) and KYSE150 (right) cells. The MTS assay was used to assess the inhibitory effect of resveratrol on ESCC cells. (B) Resveratrol suppresses anchorage-independent growth in human ESCC cells, including (a) KYSE510, (b) Eca109 and (c) KYSE150 cells. MTS and colony formation assays were conducted to investigate the viability and colony formation ability of human ESCC cells treated with various concentrations of resveratrol, compared with ESCC cells treated with DMSO. Each experiment was conducted in triplicate and independently. The data are presented as the mean \pm standard deviation. ${ }^{*} \mathrm{P}<0.05,{ }^{* *} \mathrm{P}<0.01,{ }^{* * * *} \mathrm{P}<0.001$ suppression of viability or colony formation by resveratrol vs. DMSO (control). ESCC, esophageal squamous cell carcinoma; DMSO, dimethyl sulfoxide; OD, optical density.

$30 \mathrm{~min}$. Stained cells were assayed and quantified using a FACSort Flow Cytometer (BD Biosciences). For apoptosis analysis, KYSE150 cells were suspended $\left(1 \times 10^{6}\right.$ cells $\left./ \mathrm{ml}\right)$ in staining buffer and $5 \mu \mathrm{l}$ Annexin $\mathrm{V}$ and propidium iodide (PI) staining solution were added to $300 \mu \mathrm{l}$ cell suspension. Following incubation of the cells for 10-15 min at room temperature in the dark, the stained cells were assayed and quantified using a FACSort Flow Cytometer.

Statistical analysis. All statistical analyses were performed using SPSS software version 13.0 (SPSS, Inc., Chicago, IL, USA). The experiments were all performed in triplicate and the data are expressed as the mean \pm standard deviation. The significant differences between the groups were assessed using a two-tailed Student's t-test. $\mathrm{P}<0.05$ was considered to indicate a statistically significant result.

\section{Results}

Resveratrol inhibits ESCC cell proliferation and anchorage-independent growth. The antitumor efficacy of resveratrol on ESCC cell lines was investigated using MTS and soft agar assays. At a low concentration $(20 \mu \mathrm{M})$, resveratrol exhibited little effect on the growth inhibition, but at higher concentrations (40-80 $\mu \mathrm{M})$, long-term $(48-72 \mathrm{~h})$ treatment with resveratrol significantly inhibited cell proliferation (Fig. 1A; $\mathrm{P}<0.05$ ). Furthermore, as presented in Fig. 1B, resveratrol may potently inhibit anchorage-independent cell growth, even at $20 \mu \mathrm{M}$ as the number of colonies that formed on the soft agar were significantly decreased; at high concentrations $(80 \mu \mathrm{M})$, almost no colonies were observed to form $(\mathrm{P}<0.05, \mathrm{P}<0.01$ and $\mathrm{P}<0.001)$. These results indicate that resveratrol has a marked antitumor efficacy in ESCC cells. 
A

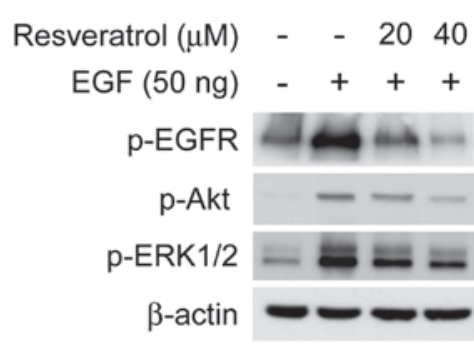

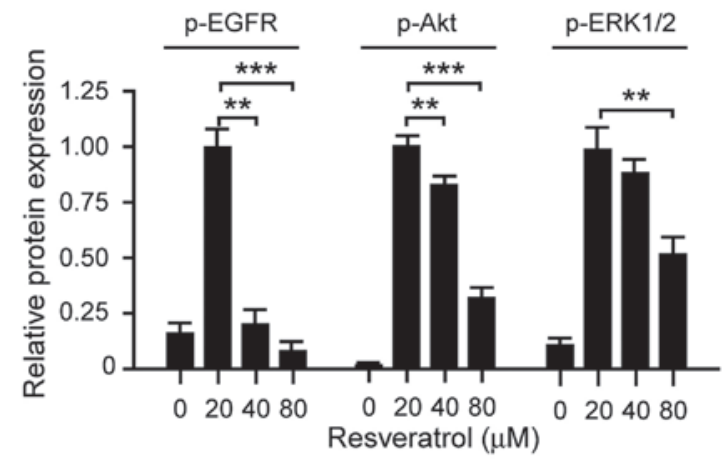

B

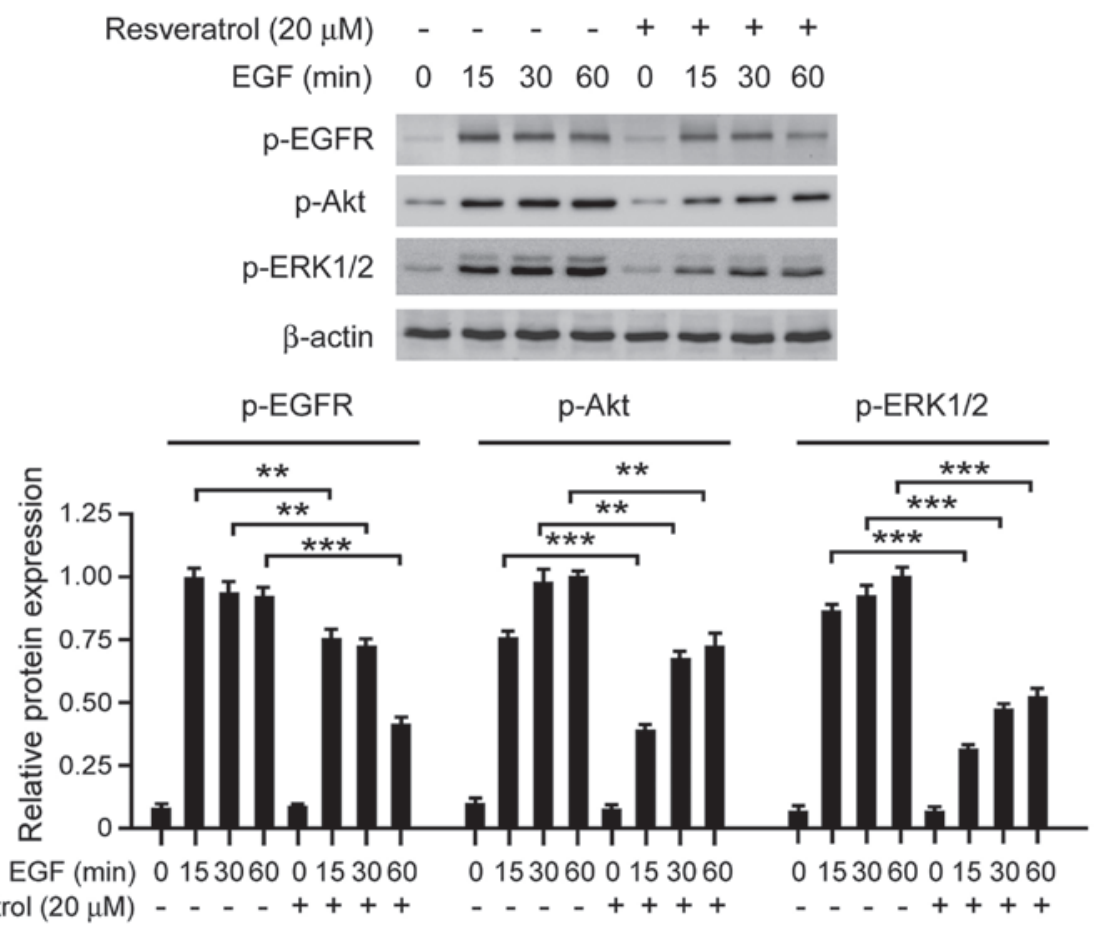

Figure 2. Resveratrol affects EGF-induced EGFR signaling in KYSE150 ESCC cells. (A) Resveratrol inhibits the EGF-induced EGFR signaling pathway in a dose-dependent manner. KYSE150 cells were treated with resveratrol, and the expression of p-EGFR, p-AKT, p-ERK1/2 and $\beta$-actin was determined by western blotting. (B) Resveratrol inhibits the EGF-induced EGFR signaling pathway in a time-dependent manner. KYSE150 cells were incubated in serum-free medium overnight. Following treatment with resveratrol for $2 \mathrm{~h}$, the cells were stimulated with EGF $(50 \mathrm{ng} / \mathrm{ml})$ for various time periods $(0,15$, 30 and $60 \mathrm{~min}$ ), p-EGFR, p-AKT, p-ERK1/2 and $\beta$-actin expression was determined by western blotting. The histograms represent the means \pm standard deviations from three independent experiments; ${ }^{* *} \mathrm{P}<0.01$ and ${ }^{* * * *} \mathrm{P}<0.001$ for suppression of protein expression by resveratrol vs. DMSO (control) group. EGF, epidermal growth factor; EGFR, epidermal growth factor receptor; ESCC, esophageal squamous cell carcinoma; p, phosphorylated; AKT, protein kinase B; ERK1/2, extracellular signal-regulated kinases $1 / 2$.

Short-term exposure to resveratrol downregulates the EGF-induced EGFR signaling pathway. The EGFR signaling pathway is frequently dysregulated in human ESCC cells or tissues and is considered to have an important role in cell proliferation and survival (22). The results of the present study revealed that treatment with resveratrol markedly inhibits EGFR activation in a dose-dependent manner (Fig. 2A). The EGF-induced phosphorylation of EGFR was markedly inhibited in KYSE150 cells by $40 \mu \mathrm{M}$ resveratrol; the phosphorylation of ERK1/2 and AKT, two downstream kinases of EGFR, were also significantly inhibited. In order to further investigate the effect of resveratrol on EGFR activation, EGF-induced EGFR phosphorylation was evaluated at various time points. Resveratrol suppressed EGFR activation, as well as its downstream kinases ERK1/2 and AKT at all selected time points (Fig. 2B). These results suggest that resveratrol exerts its antitumor effects via inhibiting EGFR activation and its downstream signaling pathways.

Long-term exposure to resveratrol downregulates EGFR protein expression in KYSE150 cells. The results of the current study demonstrated that the EGFR signaling pathway is inhibited following transient resveratrol treatment, which indicated that resveratrol may suppress the tyrosine kinase activity of EGFR in a dose-dependent manner. As presented in Fig. 3A, long-term exposure ( $>48 \mathrm{~h})$ to resveratrol decreased the expression levels of EGFR in dose-dependent manner. Following treatment with $80 \mu \mathrm{M}$ resveratrol for $48 \mathrm{~h}$, EGFR protein expression levels in KYSE150 cells were significantly downregulated. As previous studies have reported that EGFR is located in the nucleus in addition to the cellular membrane, subcellular separation was performed in order to investigate the effect of resveratrol on 
A Resveratrol $(\mu \mathrm{M}) \quad 0 \quad 20 \quad 40 \quad 80$

$$
\text { EGFR }
$$

$\beta$-actin

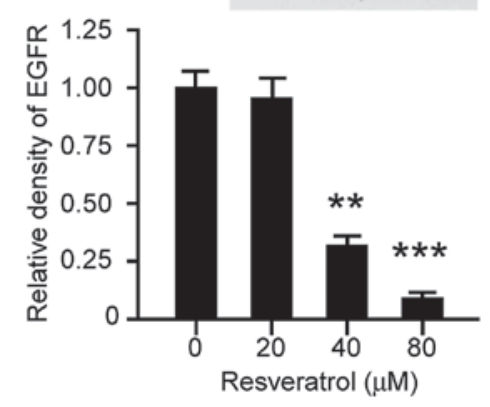

B
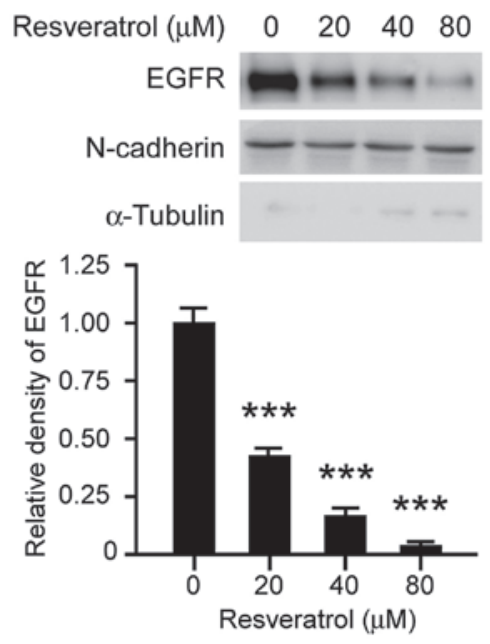

C Resveratrol $(\mu \mathrm{M}) \quad 0 \quad 20 \quad 40 \quad 80$

$$
\text { EGFR }
$$

LaminB

$\alpha$-Tubulin

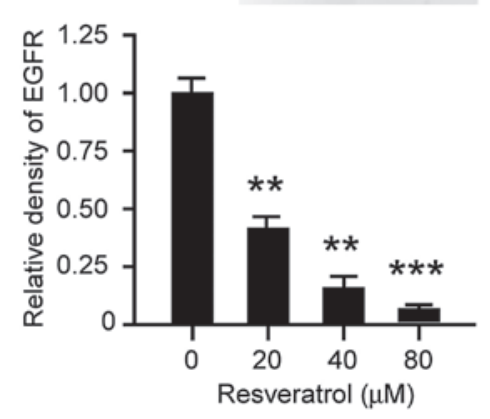

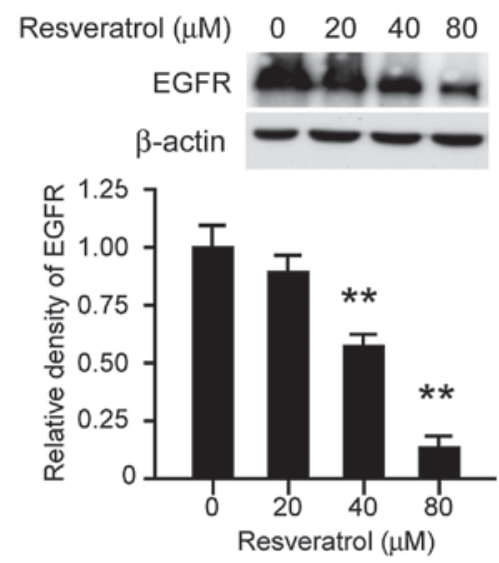
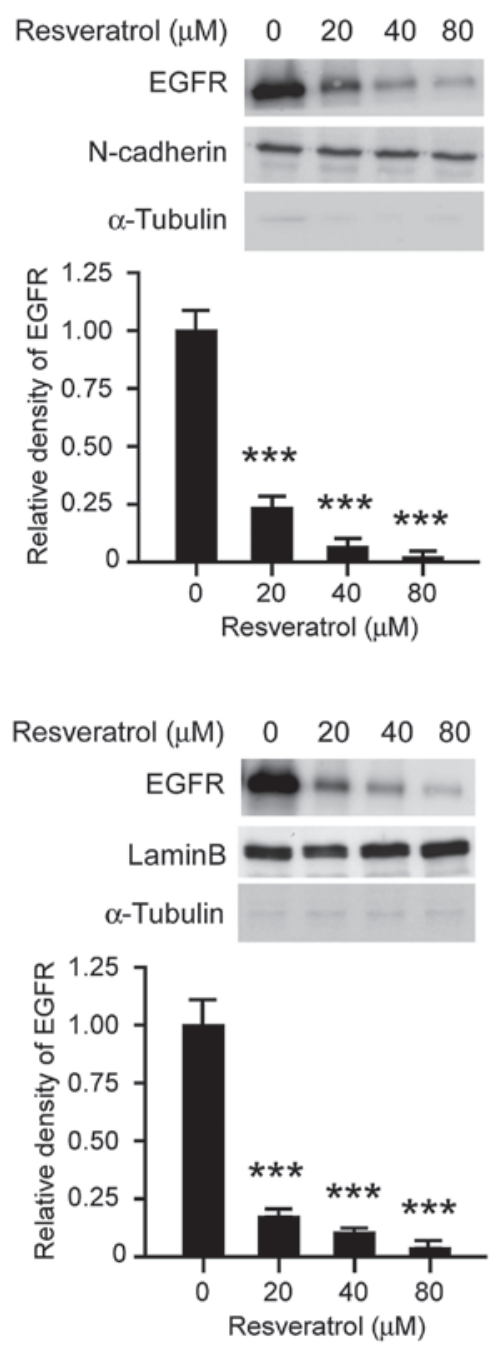

Figure 3. Resveratrol decreases the expression levels of EGFR in human ESCC cells. (A) Resveratrol suppresses EGFR expression levels in KYSE150 and Eca109 cells. KYSE150 (left) and Eca109 (right) cells were treated with various concentrations of resveratrol for 48 h. The whole cell lysates were then extracted and the protein expression levels were determined by western blot analysis. (B) Resveratrol decreases the expression levels of membrane-associated EGFR, KYSE150 (left) and Eca109 (right) cells were treated with various concentrations of resveratrol for 48 h, EGFR, N-cadherin and $\alpha$-tubulin were analyzed by western blotting. (C) Resveratrol inhibits EGFR localization to the nucleus. KYSE150 (left) and Eca109 (right) cells were treated with various concentrations of resveratrol for $48 \mathrm{~h}$, EGFR, lamin B and $\alpha$-tubulin were analyzed by western blotting. The histograms represent the means \pm standard deviations from three independent experiments; ${ }^{* *} \mathrm{P}<0.01$ and ${ }^{* * *} \mathrm{P}<0.001$ for suppression of protein expression by resveratrol vs. DMSO (control) group. EGFR, epidermal growth factor receptor; ESCC, esophageal squamous cell carcinoma.

subcellular EGFR expression patterns. As presented in Fig. 3B, membrane-associated EGFR expression levels were markedly decreased following treatment with resveratrol treated for $48 \mathrm{~h}$, in KYSE150 (left) and Eca109 (right) cells. Furthermore, the expression levels of EGFR localized to the nucleus were also downregulated following resveratrol exposure for $48 \mathrm{~h}$ (Fig. 3C). 


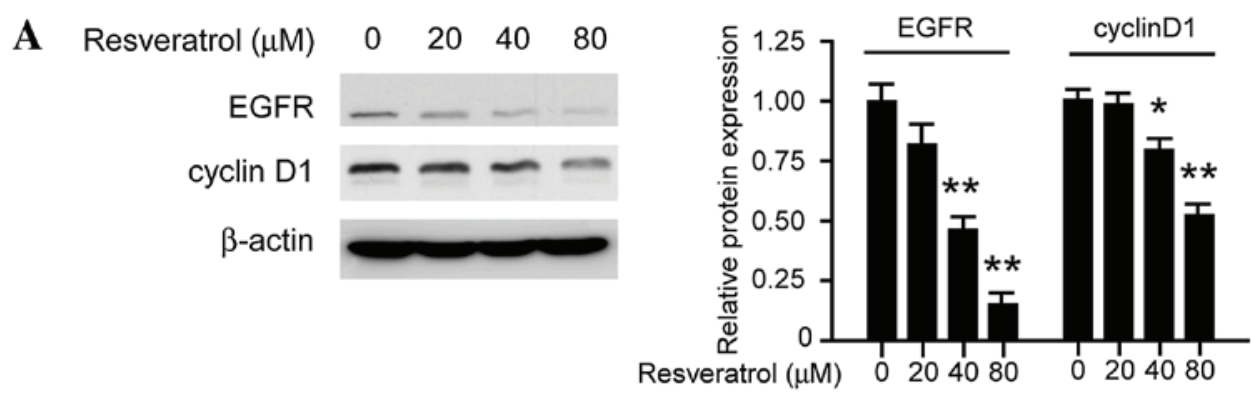

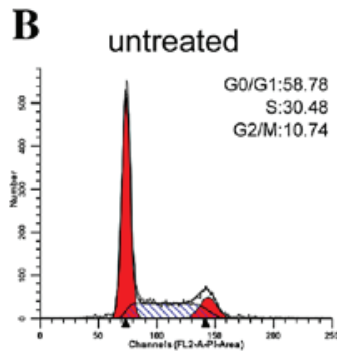

Resveratrol: $40 \mu \mathrm{M}$

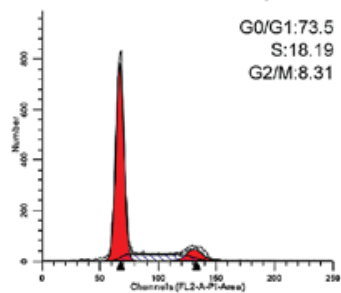

Resveratrol: $20 \mu \mathrm{M}$

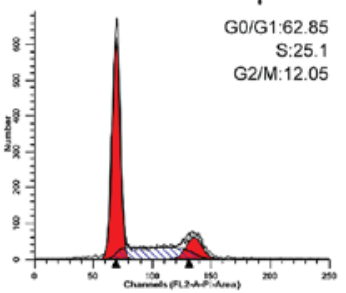

Resveratrol: $80 \mu \mathrm{M}$

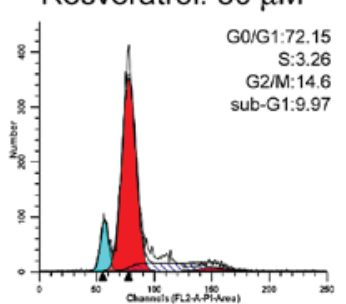

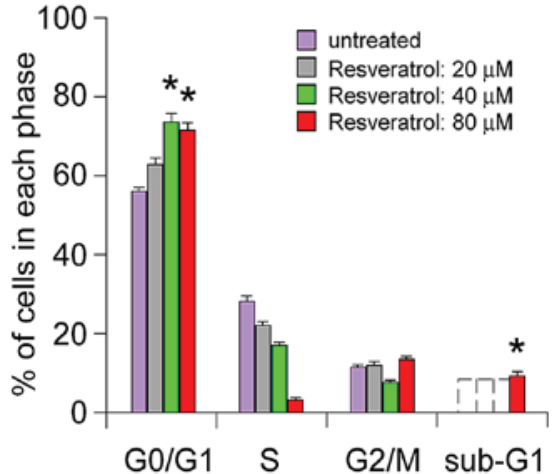

Figure 4. Resveratrol regulates cyclin D1 expression and cell cycle progression in KYSE150 cells. (A) Resveratrol dose-dependently inhibits cyclin D1 expression levels in KYSE150 cells. KYSE150 cells were treated with various concentrations of resveratrol for $24 \mathrm{~h}$. The whole cell lysates were the extracted and subjected to western blot analysis. The histograms represent the means \pm standard deviations from three independent experiments; ${ }^{*} \mathrm{P}<0.05$ and ${ }^{* *} \mathrm{P}<0.01$ for suppression of protein expression by resveratrol vs. DMSO (control) group. (B) Resveratrol dose-dependently induces cell cycle arrest at the $\mathrm{G}_{0} / \mathrm{G}_{1}$ phase. Equal numbers of KYSE150 cells in 6-well plates were treated with various concentrations of resveratrol for $24 \mathrm{~h}$ and the cell cycle analysis was conducted using flow cytometry. Each experiment was conducted in triplicate and independently. The data are presented as the mean \pm standard deviation. ${ }^{*} \mathrm{P}<0.05$, increase in the number of resveratrol treated KYSE150 cells in the $\mathrm{G}_{0} / \mathrm{G}_{1}$ phase vs. the control. EGFR, epidermal growth factor receptor.

These results suggest that, following long-term treatment with resveratrol, the expression levels of EGFR at the cell membrane and in the nucleus were suppressed.

Resveratrol suppresses cyclin D1 expression and induces cell cycle arrest at $G_{0} / G_{I}$ phase. As an important downstream target of EGFR, cyclin D1 has a crucial role in $\mathrm{G}_{1} / \mathrm{S}$ progression in the cell cycle. It was demonstrated that treatment with resveratrol for $24 \mathrm{~h}$ markedly decreased the expression levels of EGFR, as well as cyclin D1 expression levels in KYSE150 cells (Fig. 4A). Additionally, resveratrol treatment had an effect on the cell cycle, inducing cell cycle arrest at the $G_{0} / G_{1}$ phase in dose-dependent manner. At a low concentration $(20 \mu \mathrm{M})$ cell cycle arrest was not observed; however, higher concentrations (40-80 $\mu \mathrm{M})$ induced $\mathrm{G}_{0} / \mathrm{G}_{1}$ phase arrest and the proportion of cells in the $G_{0} / G_{1}$ phase was significantly increased $(P<0.05)$. As shown in Fig. $4 \mathrm{~B}, 80 \mu \mathrm{M}$ resveratrol is able to induce the sub- $\mathrm{G}_{1}$ peak, which indicated that high doses of resveratrol may directly induce cell death. The results suggest that resveratrol inhibits cyclin D1 expression levels and induces cell cycle arrest at the $\mathrm{G}_{0} / \mathrm{G}_{1}$ phase.

Resveratrol promotes cell death in KYSE150 cells. In order to further investigate the effect of resveratrol on cell death, the expression levels of apoptosis-associated molecules, including cleaved poly (ADP-ribose) polymerase (PARP) and cleaved caspase-3, were evaluated following treatment with resveratrol. Western blot analysis revealed that the expression levels of cleaved PARP and cleaved caspase-3 were markedly increased in a time-dependent manner following treatment with $80 \mu \mathrm{M}$ resveratrol (Fig. 5A). In addition, PI/Annexin V staining and flow cytometric analysis indicated that resveratrol induced KYSE150 cell death in a time-dependent manner (Fig. 5B), concordant with the results from the western blotting. Notably, it was identified that high-dose resveratrol treatment induced other types of cell death as well as apoptosis; The PI-stained cells were significantly upregulated $(\mathrm{P}<0.05)$ following resveratrol treatment. The results suggest that resveratrol treatment induces cell death in human ESCC cells.

\section{Discussion}

Numerous studies previously reported that resveratrol exhibited a marked efficacy in various types of tumor tissues, including hepatocellular carcinoma, ovarian cancer and breast cancer $(16,17)$. However, its activity and underlying mechanisms in ESCC have yet to be elucidated. In the present study, it was demonstrated that resveratrol 
A

Resveratrol (h) $0 \quad 24 \quad 4872$

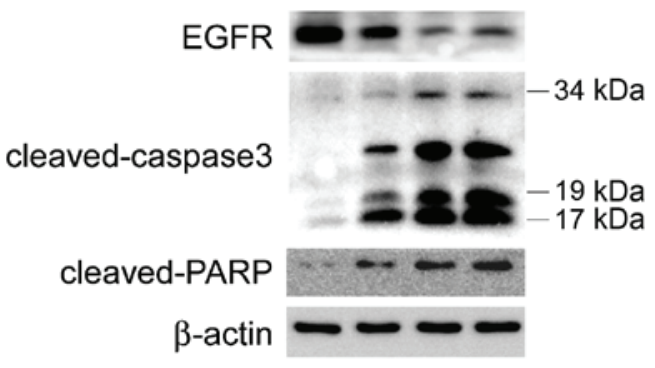

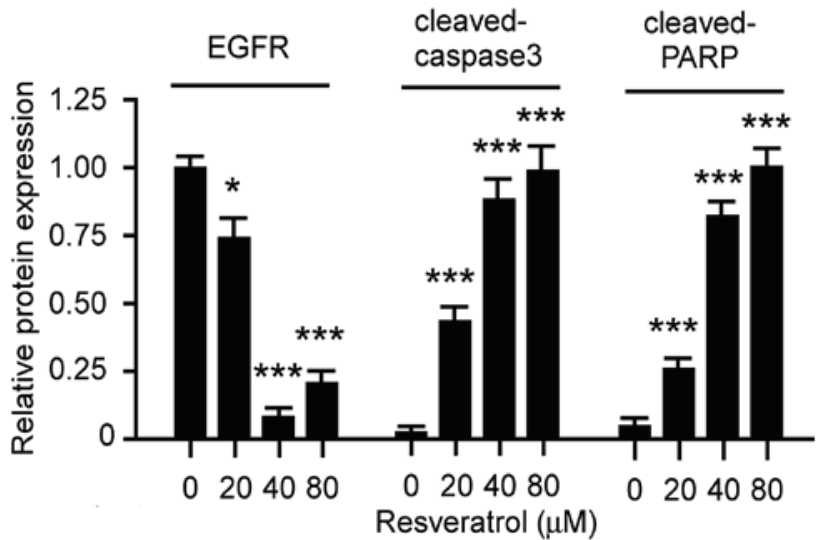

Resveratrol: $24 \mathrm{~h}$
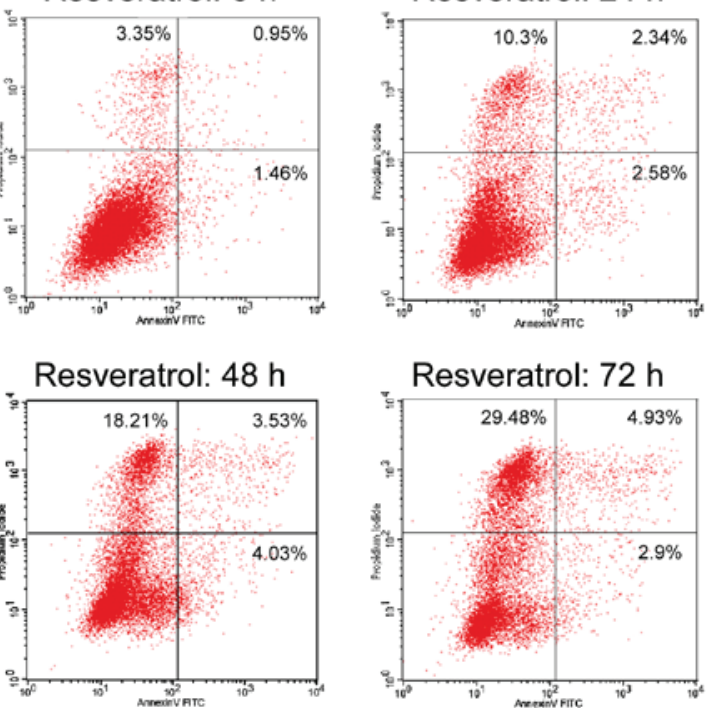

Resveratrol: $72 \mathrm{~h}$

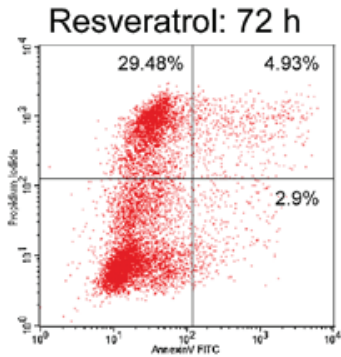

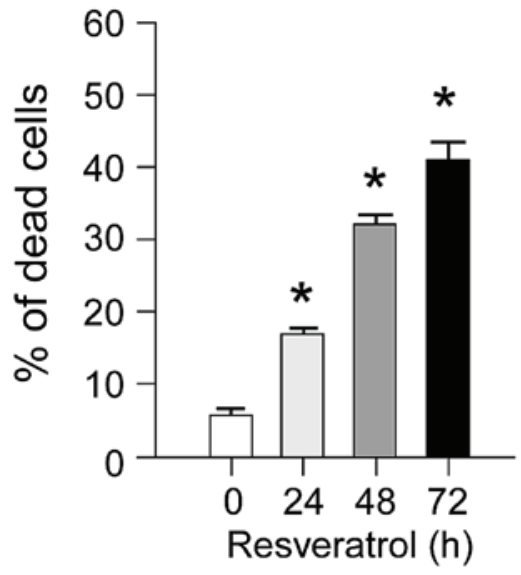

Figure 5. Resveratrol induces cell death in KYSE150 cells. (A) Resveratrol time-dependently increases cleaved PARP and cleaved caspase-3 expression levels in KYSE150 cells. KYSE150 cells were treated with $80 \mu \mathrm{M}$ resveratrol at various time points. The whole cell lysates were then extracted and subjected to western blot analysis. The histograms represent the means \pm standard deviations from three independent experiments; ${ }^{*} \mathrm{P}<0.05$ and ${ }^{* * *} \mathrm{P}<0.001$ for suppression of protein expression by resveratrol vs. DMSO (control) group. (B) Resveratrol time-dependently increases the rate of cell death in KYSE150 cells. Equal numbers of KYSE150 cells in 6-well plates were treated with $80 \mu \mathrm{M}$ resveratrol for $0,24,48$ or $72 \mathrm{~h}$. Annexin V-FITC/PI double staining and flow cytometry analysis were used to evaluate resveratrol-induced cell death in KYSE150 cells. Each experiment was conducted in triplicate and independently. The data are presented as the mean \pm standard deviation. ${ }^{*} \mathrm{P}<0.05$, increase in resveratrol-induced cell death vs. the control. FITC, fluorescein isothiocyanate; PI, propidium iodide; EGFR, epidermal growth factor receptor; PARP, poly (ADP-ribose) polymerase.

dose-dependently inhibits ESCC cell proliferation in vitro and that colony formation is markedly decreased following resveratrol treatment (Fig. 1). Furthermore, exposure of ESCC cells to resveratrol effectively induced $\mathrm{G}_{0} / \mathrm{G}_{1}$ cell cycle arrest. In addition, resveratrol treatment stimulated ESCC cells to undergo apoptosis, as revealed by flow cytometry and western blot analysis. Further investigation revealed that resveratrol exerts its antitumor activity by regulating the EGFR signaling pathway in ESCC.

EGFR has an important role in tumor development, including in cell proliferation, anti-apoptosis, angiogenesis, tumor metastasis and invasion $(23,24)$. Dysregulation of the EGFR signaling pathway through overexpression or hyperactivation is associated with tumor development $(23,25,26)$. A meta-analysis demonstrated that the rate of EGFR overexpression in ESCC is high and EGFR expression levels are a potential predictor of the $\mathrm{T}$ stage (tumor-node-metastasis classification system) (27), vascular invasion and overall survival (27). By contrast, Aichler et al (28) reported that EGFR is an independent adverse prognostic factor in esophageal adenocarcinoma patients treated with cisplatin-based neoadjuvant chemotherapy; therefore, targeting EGFR may be a novel therapeutic approach in the management of ESCC. EGFR is typically expressed at the cellular membrane, and is activated via autophosphorylation on binding with its ligands, epidermal growth factor (EGF) or transforming growth factor- $\alpha$. EGFR phosphorylation activates numerous downstream signaling pathways, including the rat sarcoma/rapidly accelerated fibrosarcoma/mitogen activated protein kinase and phosphoinositide-3-kinase/AKT signaling pathways, which regulate various cellular functions $(29,30)$. In the present study, the results demonstrated that short-term exposure to resveratrol significantly inhibits EGF-induced EGFR phosphorylation. Following the inhibition of EGFR activation, the downstream signaling pathways and phosphorylation of EGFR, ERK and AKT were suppressed. 
Similarly to a number of natural compounds, including epigallocatechin gallate (31) and quercetin (32), resveratrol has also been demonstrated to inhibit various kinases (33). The current study only investigated the effect of resveratrol on the EGFR signaling pathway in human ESCC cells, which is one limitation as resveratrol may also target other kinases in ESCC cells. It was also identified that long-term $(48 \mathrm{~h})$ exposure to resveratrol in ESCC cells induces EGFR degradation. Previous studies have demonstrated that the phosphorylation of EGFR at Tyr1045 creates a docking site for casitas B-lineage lymphoma, an adaptor protein that leads to receptor ubiquitination and degradation following EGFR activation $(33,34)$. It is possible that resveratrol may induce EGFR degradation via the ubiquitination or lysosome signaling pathways in human ESCC cells. Further investigation revealed that the expression levels of EGFR at the cell membrane were reduced and EGFR located in the nucleus was also significantly decreased following resveratrol treatment. It was hypothesized that reduction of EGFR expression levels at the cell membrane may reduce the sensitivity of the cell to growth factors, and cell proliferation may be markedly decreased following stimulation with growth factors.

Previous studies have demonstrated that the nuclear import of EGFR and the signal cascade induced by nuclear EGFR had pivotal roles in the malignant proliferation and survival of certain types of tumor tissues (35-37). Immunohistochemical analysis of breast tumor tissues revealed that EGFR expression levels in the nucleus are positively correlated with the expression of Ki-67, a biomarker of cell proliferation, and negatively correlated with the survival rate $(38,39)$. In oral squamous cell carcinoma (OSCC), elevated nuclear import of EGFR may predict a poor outcome for OSCC patients (40). It has also been identified that nuclear EGFR is involved in acquired chemotherapy resistance (41). In pancreatic cancer, nucleus EGFR combines with sarcoma and signal transducer and activator of transcription 5 to form a complex that regulates the transcription of the $c$-myc gene and to mediates the sensitivity of tumor cells to chemotherapy (42). Previous studies also identified that EGFR nuclear import upregulated the activity of DNA-dependent protein kinases (DNA-PK) and promoted the repair of DNA damage (43). The EGFR monoclonal antibody Cetuximab may block EGFR nuclear import, downregulate the activity of DNA-PK, inhibit DNA repair and induce tumor cell apoptosis (43-46). The present study demonstrated that long-term treatment with resveratrol markedly decreases the expression levels of EGFR in the nucleus, suggesting that resveratrol may assist the prevention of chemotherapy resistance when combined with cytotoxic drugs.

In conclusion, the results demonstrated that resveratrol is effective in inhibiting the proliferation of ESCC cells, suggesting that a dietary pattern rich in resveratrol may reduce the risk of ESCC, as initially hypothesized by Lin et al (15). The results also revealed that resveratrol influences EGFR activity in ESCC cells, short-term exposure of ESCC cells to resveratrol had an inhibitory effect on EGFR phosphorylation and its signaling pathway, but long-term treatment induced EGFR degradation and had an effect on the activity of EGFR in the nucleus and at the cell membrane. The results suggested that resveratrol, or analog, may have a role in the prevention and management of ESCC.

\section{Acknowledgements}

This study was supported by the National Nature Science Foundation of China (grant no. 81472774).

\section{References}

1. van Hagen P, Hulshof MC, van Lanschot JJ, Steyerberg EW, van Berge Henegouwen MI, Wijnhoven BP, Richel DJ, Nieuwenhuijzen GA, Hospers GA, Bonenkamp JJ, et al: Preoperative chemoradiotherapy for esophageal or junctional cancer. N Engl J Med 366: 2074-2084, 2012.

2. Enzinger PC and Mayer RJ: Esophageal cancer. N Engl J Med 349: 2241-2252, 2003.

3. Hongo M, Nagasaki Y and Shoji T: Epidemiology of esophageal cancer: Orient to Occident. Effects of chronology, geography and ethnicity. J Gastroenterol Hepatol 24: 729-735, 2009.

4. Cheung WY and Liu G: Genetic variations in esophageal cancer risk and prognosis. Gastroenterol Clin North Am 38: 75-91, viii, 2009.

5. Wu C, Hu Z, He Z, Jia W, Wang F, Zhou Y, Liu Z, Zhan Q, Liu Y, Yu D, et al: Genome-wide association study identifies three new susceptibility loci for esophageal squamous-cell carcinoma in Chinese populations. Nat Genet 43: 679-684, 2011.

6. Wu C, Li D, Jia W, Hu Z, Zhou Y, Yu D, Tong T, Wang M, Lin D, Qiao Y, et al: Genome-wide association study identifies common variants in SLC39A6 associated with length of survival in esophageal squamous-cell carcinoma. Nat Genet 45: 632-638, 2013

7. Gao YB, Chen ZL, Li JG, Hu XD, Shi XJ, Sun ZM, Zhang F, Zhao ZR, Li ZT, Liu ZY, et al: Genetic landscape of esophageal squamous cell carcinoma. Nat Genet 46: 1097-1102, 2014.

8. Gescher A, Steward WP and Brown K: Resveratrol in the management of human cancer: How strong is the clinical evidence? Ann N Y Acad Sci 1290: 12-20, 2013.

9. Frazzi R and Tigano M: The multiple mechanisms of cell death triggered by resveratrol in lymphoma and leukemia. Int J Mol Sci 15: 4977-4993, 2014.

10. Park EJ and Pezzuto JM: The pharmacology of resveratrol in animals and humans. Biochim Biophys Acta 1852: 1071-1113, 2015.

11. Novelle MG, Wahl D, Diéguez C, Bernier M and de Cabo R: Resveratrol supplementation: Where are we now and where should we go? Ageing Res Rev 21: 1-15, 2015.

12. Singh CK, George J and Ahmad N: Resveratrol-based combinatorial strategies for cancer management. Ann N Y Acad Sci 1290: 113-121, 2013.

13. Xue YQ, Di JM, Luo Y, Cheng KJ, Wei X and Shi Z: Resveratrol oligomers for the prevention and treatment of cancers. Oxid Med Cell Longev 2014: 765832, 2014.

14. Yang X, Li X and Ren J: From French Paradox to cancer treatment: Anti-cancer activities and mechanisms of resveratrol. Anticancer Agents Med Chem 14: 806-825, 2014.

15. Lin Y, Yngve A, Lagergren J and Lu Y: A dietary pattern rich in lignans, quercetin and resveratrol decreases the risk of oesophageal cancer. Br J Nutr 112: 2002-2009, 2014.

16. Kulkarni SS and Cantó C: The molecular targets of resveratrol. Biochim Biophys Acta 1852: 1114-1123, 2015.

17. Singh CK, Ndiaye MA and Ahmad N: Resveratrol and cancer: Challenges for clinical translation. Biochim Biophys Acta 1852: 1178-1185, 2015.

18. Britton RG, Kovoor C and Brown K: Direct molecular targets of resveratrol: Identifying key interactions to unlock complex mechanisms. Ann N Y Acad Sci 1348: 124-133, 2015.

19. Tebbutt N, Pedersen MW and Johns TG: Targeting the ERBB family in cancer: Couples therapy. Nat Rev Cancer 13: 663-673, 2013

20. Hanawa M, Suzuki S, Dobashi Y, Yamane T, Kono K, Enomoto $\mathrm{N}$ and Ooi A: EGFR protein overexpression and gene amplification in squamous cell carcinomas of the esophagus. Int J Cancer 118: 1173-1180, 2006.

21. Fichter CD, Gudernatsch V, Przypadlo CM, Follo M, Schmidt $G$, Werner $M$ and Lassmann S: ErbB targeting inhibitors repress cell migration of esophageal squamous cell carcinoma and adenocarcinoma cells by distinct signaling pathways. J Mol Med (Berl) 92: 1209-1223, 2014. 
22. Hoshino M, Fukui H, Ono Y, Sekikawa A, Ichikawa K, Tomita S, Imai Y, Imura J, Hiraishi H and Fujimori T: Nuclear expression of phosphorylated EGFR is associated with poor prognosis of patients with esophageal squamous cell carcinoma. Pathobiology 74: 15-21, 2007.

23. Mitsudomi T and Yatabe Y: Epidermal growth factor receptor in relation to tumor development: EGFR gene and cancer. FEBS J 277: 301-308, 2010

24. Olayioye MA, Neve RM, Lane HA and Hynes NE: The ErbB signaling network: Receptor heterodimerization in development and cancer. EMBO J 19: 3159-3167, 2000.

25. Han W and Lo HW: Landscape of EGFR signaling network in human cancers: Biology and therapeutic response in relation to receptor subcellular locations. Cancer Lett 318: 124-134, 2012.

26. Kolch W and Pitt A: Functional proteomics to dissect tyrosine kinase signalling pathways in cancer. Nat Rev Cancer 10 $618-629,2010$

27. Wang J, Yu JM, Jing SW, Guo Y, Wu YJ, Li N, Jiao WP, Wang L and Zhang YJ: Relationship between EGFR over-expression and clinicopathologic characteristics in squamous cell carcinoma of the esophagus: A meta-analysis. Asian Pac J Cancer Prev 15: 5889-5893, 2014

28. Aichler M, Motschmann M, Jutting U, Luber B, Becker K, Ott K, Lordick F, Langer R, Feith M, Siewert JR and Walch A Epidermal growth factor receptor (EGFR) is an independent adverse prognostic factor in esophageal adenocarcinoma patients treated with cisplatin-based neoadjuvant chemotherapy. Oncotarget 5: 6620-6632, 2014.

29. Gan HK, Burgess AW, Clayton AH and Scott AM: Targeting of a conformationally exposed, tumor-specific epitope of EGFR as a strategy for cancer therapy. Cancer Res 72: 2924-2930, 2012.

30. Lacouture ME: Mechanisms of cutaneous toxicities to EGFR inhibitors. Nat Rev Cancer 6: 803-812, 2006.

31. Schramm L: Going Green: The role of the green tea component EGCG in chemoprevention. J Carcinog Mutagen 4: 1000142, 2013.

32. Russo GL, Russo M, Spagnuolo C, Tedesco I, Bilotto S, Iannitti R and Palumbo R: Quercetin: A pleiotropic kinase inhibitor against cancer. Cancer Treat Res 159: 185-205, 2014.

33. Levkowitz G, Waterman H,Ettenberg SA, Katz M, Tsygankov AY, Alroy I, Lavi S, Iwai K, Reiss Y, Ciechanover A, et al: Ubiquitin ligase activity and tyrosine phosphorylation underlie suppression of growth factor signaling by c-Cbl/Sli-1. Mol Cell 4: 1029-1040, 1999.

34. Ettenberg SA, Keane MM, Nau MM, Frankel M, Wang LM, Pierce JH and Lipkowitz S: cbl-b inhibits epidermal growth factor receptor signaling. Oncogene 18: 1855-1866, 1999.
35. Lin SY, Makino K, Xia W, Matin A, Wen Y, Kwong KY, Bourguignon L and Hung MC: Nuclear localization of EGF receptor and its potential new role as a transcription factor. Nat Cell Biol 3: 802-808, 2001.

36. Brand TM, Iida M, Luthar N, Starr MM, Huppert EJ and Wheeler DL: Nuclear EGFR as a molecular target in cancer. Radiother Oncol 108: 370-377, 2013.

37. Li A, Zhang C, Gao S, Chen F, Yang C, Luo R and Xiao H: TIP30 loss enhances cytoplasmic and nuclear EGFR signaling and promotes lung adenocarcinogenesis in mice. Oncogene 32 2273-2281, 2281e.1-12, 2013.

38. Umemura S, Takekoshi S, Suzuki Y, Saitoh Y, Tokuda Y and Osamura RY: Estrogen receptor-negative and human epidermal growth factor receptor 2-negative breast cancer tissue have the highest $\mathrm{Ki}-67$ labeling index and EGFR expression: Gene amplification does not contribute to EGFR expression. Oncol Rep 14 337-343, 2005.

39. Lo HW, Xia W, Wei Y, Ali-Seyed M, Huang SF and Hung MC Novel prognostic value of nuclear epidermal growth factor receptor in breast cancer. Cancer Res 65: 338-348, 2005.

40. Taguchi T: Nuclear translocation of epidermal growth factor receptor and its relation to clinicopathological factors in oral squamous cell carcinomas. Kokubyo Gakkai Zasshi 81: 45-52, 2014 (In Japanese)

41. Lo HW: Nuclear mode of the EGFR signaling network: Biology, prognostic value, and therapeutic implications. Discov Med 10: 44-51, 2010.

42. Jaganathan S, Yue P, Paladino DC, Bogdanovic J, Huo Q and Turkson J: A functional nuclear epidermal growth factor receptor, SRC and Stat 3 heteromeric complex in pancreatic cancer cells. PLoS One 6: e19605, 2011.

43. Dittmann K, Mayer C and Rodemann HP: Inhibition of radiation-induced EGFR nuclear import by C225 (Cetuximab) suppresses DNA-PK activity. Radiother Oncol 76: 157-161, 2005.

44. Dittmann K, Mayer C, Wanner G, Kehlbach R and Rodemann HP. The radioprotector O-phospho-tyrosine stimulates DNA-repair via epidermal growth factor receptor- and DNA-dependent kinase phosphorylation. Radiother Oncol 84: 328-334, 2007.

45. Liccardi G, Hartley JA and Hochhauser D: Importance of EGFR/ERCC1 interaction following radiation-induced DNA damage. Clin Cancer Res 20: 3496-3506, 2014.

46. Dittmann K, Mayer C, Kehlbach R and Rodemann HP: Radiation-induced caveolin-1 associated EGFR internalization is linked with nuclear EGFR transport and activation of DNA-PK. Mol Cancer 7: 69, 2008 